\section{Number of Benzenoid Hydrocarbons}

\section{Ivan Gutman}

Faculty of Science, University of Kragujevac, Yugoslavia

Z. Naturforsch. 41 a, 1089-1090 (1986); received July 5, 1986

The number of benzenoid hydrocarbons with $h$ hexagons can be estimated by means of the formula $B_{\mathrm{h}}=$ $0.045 h^{-3 / 2}(5.4)^{h}$. The analogous estimate for the number of catacondensed benzenoids is $C_{\mathrm{h}}=0.049 h^{-5 / 4}(4.27)^{h}$.

\section{Introduction}

The problem of the enumeration of alkanes and related chemical compounds has been solved by Pólya long time ago $[1,2]$. Another problem of this kind, namely the enumeration of benzenoid hydrocarbons, seems to be much more difficult and was not satisfactorily solved so far. In spite of serious attempts of a number of mathematicians [3-6], at the present moment we do not know anything better than to construct the benzenoids and then to count them. Several approaches along these lines have been elaborated [7-15], usually based on an extensive use of computers.

In the following we shall be interested in geometrically planar, simply connected benzenoids [16]. The number of such systems, possessing $h$ hexagons will be denoted by $B_{h}$. In addition to this, $C_{h}$ is the number of geometrically planar catacondensed benzenoids [16] with $h$ hexagons. The numbers $B_{h}$ and $C_{h}$ are nowadays known for $h \leqq 11$ and are given in Tables 1 and 2 .

For small values of $h, B_{h}$ and $C_{h}$ can be obtained without difficulty. For $h$ up to $10, B_{h}$ and $C_{h}$ were first reported by Knop et al. [11,12], whereas $B_{11}$ and $C_{11}$ were recently calculated by Doroslovački and Tošić [15]. The amount of computing, required for the evaluation of $B_{h}$ for $h \geqq 10$ is enormous and increases very rapidly with the increasing number of hexagons. Therefore, even when quite powerful computing machines have been employed, the enumeration procedure could not exceed $h=10[11,14]$ and $h=11$ [15].

These difficulties motivated us to develop approximate expressions for $B_{h}$ and $C_{h}$ which enable the estimation of these numbers for large (greater than eleven) values of $h$.

Asymptotic expressions for the estimation of the number of combinatorial objects of certain types are often met in the theory of enumeration [17]. Already Pólya [1] deduced such a formula for the number of alkanes. In a great number of cases [17], the asymptotes have the form

$$
X_{n} \sim a n^{p} b^{n} ; \quad n \rightarrow \infty,
$$

where $a$ and $b$ are some constants and the exponent $p$ is a rational number.

Reprint requests to Prof. Dr. Ivan Gutman, Faculty of Science, P.O. Box 60, YU-34000 Kragujevac, Yugoslavia.
In particular, Harary and Read [5] showed that for large values of $h$,

$$
H_{h} \sim \sqrt{\frac{5}{4}} \frac{(2 h-1) !}{(h-1) !(h+2) !}\left(\frac{5}{4}\right)^{h},
$$

where $H_{h}$ counts the catacondensed benzenoids (both geometrically planar and non-planar), with $h$ hexagons. Using the Stirling approximation, we can transform (2) into

$$
H_{h} \sim \sqrt{\frac{5}{16 \pi}} h^{-5 / 2} 5^{h},
$$

which is just a special case of (1).

\section{An Approximate Asymptote for $C_{h}$}

Bearing in mind the result (3), we considered the formula

$$
C_{h} \sim a h^{p} b^{h},
$$

which is expected to hold for sufficiently large values of $h$. In order to determine the exponent $p$ we have calculated the expression

$$
b_{h}=\left(\frac{h}{h+1}\right)^{p} \frac{C_{h+1}}{C_{h}}
$$

for the known $C_{h}$ 's (see Table 1). If $C_{h}$ behaves according to (4), then for a properly chosen $p$ the sequence $b_{1}, b_{2}, b_{3}$, $\ldots$ will rapidly converge to its limit value $b$. By varying $p$ we found that the best convergence occurs for $p=-5 / 4$. (As a matter of fact, the choice $p=-1.25$ is better than $p=-1.24$ or $p=1.26$.) The last calculated members of the sequence $b_{h}$, for $p=-5 / 4$ are given as

\begin{tabular}{rl}
\hline$h$ & $b_{h}$ \\
\hline 7 & 4.116 \\
8 & 4.198 \\
9 & 4.26887 \\
10 & 4.26895,
\end{tabular}

from which we conclude that the limiting value of $b_{h}$ is about 4.27 .

It remains to determine the parameter $a$ as the limit of the sequence $a_{1}, a_{2}, a_{3}, \ldots$, where

$$
a_{h}=C_{h} /\left(h^{p} b^{h}\right) \text {. }
$$

The fact that for $p=-5 / 4$ and $b=4.27$,

\begin{tabular}{rl}
\hline$h$ & $a_{h}$ \\
\hline 8 & 0.0500 \\
9 & 0.049187 \\
10 & 0.049174 \\
11 & 0.049162
\end{tabular}

implies $a=0.049$.

0340-4811/86/0800-1089 \$01.30/0. - Please order a reprint rather than making your own copy. 
Table 1. Exact and estimated values for the number $C_{h}$ of geometrically planar catacondensed benzenoid systems with $h$ hexagons.

Table 2. Exact and estimated values for the number $B_{h}$ of geometrically planar, simply connected benzenoid systems with $h$ hexagons.

Table 1

\begin{tabular}{lll}
\hline$h \quad C_{h}$ & $\begin{array}{l}\text { Estimate } \\
(5)\end{array}$ \\
\hline
\end{tabular}

\begin{tabular}{rrr}
\hline 1 & 1 & 0 \\
2 & 1 & 0 \\
3 & 2 & 1 \\
4 & 5 & 3 \\
5 & 12 & 9 \\
6 & 36 & 32 \\
7 & 118 & 111 \\
8 & 411 & 402 \\
9 & 1489 & 1483 \\
10 & 5572 & 5552 \\
11 & 21115 & 21046 \\
12 & & 80604 \\
13 & & 311408 \\
14 & & 1212066 \\
15 & & 4747884 \\
\hline
\end{tabular}

Table 2

\begin{tabular}{|c|c|c|}
\hline$h$ & $B_{h}$ & $\begin{array}{l}\text { Estimate } \\
\text { (6) }\end{array}$ \\
\hline 1 & 1 & 0 \\
\hline 2 & 1 & 0 \\
\hline 3 & 3 & 1 \\
\hline 4 & 7 & 5 \\
\hline 5 & 22 & 18 \\
\hline 6 & 81 & 76 \\
\hline 7 & 331 & 325 \\
\hline 8 & 1435 & 1438 \\
\hline 9 & 6505 & 6507 \\
\hline 10 & 30086 & 30002 \\
\hline 11 & 141229 & 140428 \\
\hline 12 & & 665527 \\
\hline 13 & & 3187251 \\
\hline 14 & & 15400439 \\
\hline 15 & & 74986317 \\
\hline
\end{tabular}

Thus we arrived at the approximate asymptotic expression

$$
C_{h} \sim 0.049 h^{-5 / 4}(4.27)^{h},
$$

whose quality can be seen from the data given in Table 1. In Table 1 we also presented the predicted (approximate) values of $C_{12}, C_{13}, C_{14}$ and $C_{15}$.

\section{An Approximate Asymptote for $\boldsymbol{B}_{h}$}

In the case of $B_{h}$, a completely analogous variational procedure gave the optimal value -1.47 for the exponent $p$, which is satisfactorily close to the adopted value $-3 / 2$. The choice $p=-3 / 2$ leads then to the numbers

\begin{tabular}{rll}
\hline$h$ & $b_{h}$ & $a_{h}$ \\
\hline 7 & 5.297 & \\
8 & 5.409 & 0.0449 \\
9 & 5.41693 & 0.044985 \\
10 & 5.41562 & 0.045126 \\
11 & - & 0.045224
\end{tabular}

which imply$$
B_{h} \sim 0.045 h^{-3 / 2}(5.4)^{h} \text {. }
$$

The exact $B_{h}$ values as well as those calculated by means of (6) are collected in Table 2, together which the estimates for $B_{12}, B_{13}, B_{14}$ and $B_{15}$. Formula (6) seems to be somewhat less accurate than (5).
[1] G. Pólya, Z. Kristal. 93 A, 415 (1936)

[2] G. Pólya, Acta Math. 68, 145 (1937).

[3] D. A. Klarner, Fibonacci Quart. 3, 9 (1965).

[4] D. A. Klarner, Canad. J. Math. 19,851 (1967).

[5] F. Harary and R. C. Read, Proc. Edinburgh Math. Soc. 17, 1 (1970).

[6] W. F. Lunnon, in: R. C. Read (Ed.), Graph Theory and Computing, Academic Press, New York 1972, pp. $87-100$.

[7] K. Balasubramanian, J. J. Kaufman, W. S. Koski, and A. T. Balaban, J. Comput. Chem. 1, 149 (1980).

[8] J. R. Dias, J. Chem. Inf. Comput. Sci. 22, 15 (1982).

[9] J. R. Dias, Match (Mülheim) 14, 83 (1983).

[10] J. R. Dias, J. Chem. Inf. Comput. Sci. 24, 124 (1984).

[11] J. V. Knop, K. Szymanski, Z. Jeričević, and N. Trinajstić, J. Comput. Chem. 4, 23 (1983).

[12] J. V. Knop, K. Szymanski, Ž. Jeričević, and N. Trinajstić, Match (Mülheim) 16,119 (1984).
[13] S. J. Cyvin and J. Brunvoll (Trondheim, Norway), private communication, Spring 1986.

[14] J. Ciosłowski (Washington, USA), private communication, Spring 1986.

[15] R. Doroslovački and R. Tošić (Novi Sad, Yugoslavia), private communication, Spring 1986.

[16] A benzenoid system is geometrically planar if its nonadjacent (regular) hexagons do not overlap. A benzenoid system is simply connected if it separates the plane into an infinite region and $h$ finite regions, all of which are (regular) hexagons. A benzenoid system is catacondensed if no three of its hexagons are mutually adjacent. More details on benzenoid systems can be found in: I. Gutman and O. E. Polansky, Mathematical Concepts in Organic Chemistry, SpringerVerlag, Berlin 1986, pp. 59-61.

[17] F. Harary and E. P. Palmer, Graphical Enumeration, Academic Press, New York 1973, Chapter 9. 